\title{
6. The affine breadth and its relation \\ to the relative breadth.
}

\author{
By Junkô HirakawA.
}

(Received June 12, 1935)

Prof. W. Süss has made an investigation concerning the affine constant breadth. $\left({ }^{1}\right)$ But I will make some considerations of the affine breadth from a different point of view.

In the following lines we use the same notations as in my preceding paper "On the Relative Breadth." $\left({ }^{2}\right)$

\section{$\S 1$. The affine breadth of the oval.}

Let us define $b$ :

$$
b=2 \frac{\left(\left(\mathfrak{x}_{2}-x_{1}\right) \xi_{1}\right)}{\left(\mathfrak{c}_{1}^{\prime \prime} \xi_{1}\right)+\left(\mathfrak{C}_{2}^{\prime \prime} \xi_{2}\right)}=2 \frac{p_{1}+p_{2}}{\bar{\rho}_{1}^{-\frac{1}{3}}+\bar{\rho}_{2}^{-\frac{1}{3}}} .
$$

as the affine breadth at the point $x$ of an oval $E(x)$.

Theorem 1. Let $\mathfrak{p}_{i}$ denote the affine distance from $\mathfrak{x}_{i}$ to $\mathfrak{x}_{k}(i, k=1,2$; $i \neq k$ ) where $\mathfrak{x}_{1}$ and $\mathfrak{x}_{2}$ are the points of contact of the parallel tangents, then $b$ is equal to the harmonic mean of $p_{1}$ and $\mathfrak{p}_{2}$ and $b$ is an affine invariant.

Proof. By assumption we have

$$
\mathfrak{p}_{1}=\bar{\rho}_{1}^{\frac{1}{3}}\left(\left(\mathfrak{x}_{2}-x_{1}\right) \xi_{1}\right), \quad \mathfrak{p}_{2}=\bar{\rho}_{2}^{\frac{1}{3}}\left(\left(\mathfrak{x}_{1}-x_{2}\right) \xi_{2}\right) \cdot\left({ }^{3}\right)
$$

From the definition of $b$ the relation follows

$$
\begin{aligned}
\frac{2}{b} & =\frac{\bar{\rho}_{1}^{-\frac{1}{3}}+\bar{\rho}_{2}^{-\frac{1}{3}}}{\left(\left(\mathfrak{c}_{2}-\mathfrak{x}_{1}\right) \xi_{1}\right)}, \\
& =\frac{1}{\bar{\rho}_{1}^{\frac{1}{3}}\left(\left(\mathfrak{c}_{2}-\mathfrak{x}_{1}\right) \xi_{1}\right)}+\frac{1}{\bar{\rho}_{2}^{\frac{1}{3}}\left(\left(\mathfrak{x}_{1}-\mathfrak{x}_{2}\right) \xi_{2}\right)}, \\
& =\frac{1}{\mathfrak{p}_{1}}+\frac{1}{\mathfrak{p}_{2}} .
\end{aligned}
$$

(1) Ůber affine Geometrie XL: Eiflächen konstanter Affinbreite; Math. Ann., 96 (1927)。

(2) Proceedings of the Physico-Math. Soc. of Japan, 17 (1935).

( ${ }^{3}$ W. Blaschke, Vorlesungen über Differentialgeometrie II. 
Theorem 2.A necessary and sufficient condition that the affine breadth of $x$ be constant is that the two vectors $\mathfrak{x}_{2}-x_{1}$ and $x_{1}^{\prime \prime}-x_{2}^{\prime \prime}$ are parallel.

Proof. From the definition of $b$ we have

$$
\left(\left\{\mathfrak{x}_{1}-x_{2}+\frac{b}{2}\left(x_{1}^{\prime \prime}-x_{2}^{\prime \prime}\right)\right\} \xi_{1}\right)=0 \text {. }
$$

Then it can be put

$$
\mathfrak{x}_{1}-\mathfrak{x}_{2}+\frac{b}{2}\left(\mathfrak{x}_{1}^{\prime \prime}-\mathfrak{x}_{2}^{\prime \prime}\right)=\sigma \mathfrak{x}_{1}^{\prime}
$$

where $\sigma$ is a scalar quantity.

If $b$ is constant, we have by differentiation of (3) with respect to the affine curve length $u_{1}$ at $x_{1}$

$$
\mathfrak{x}_{1}^{\prime}-\mathfrak{x}_{2}^{\prime} \frac{d u_{2}}{d u_{1}}+\frac{b}{2}\left(\mathfrak{x}_{1}^{\prime \prime \prime}-\mathfrak{x}_{2}^{\prime \prime \prime} \frac{d u_{2}}{d u_{1}}\right)=\sigma \mathfrak{x}_{1}^{\prime \prime}+\sigma^{\prime} \mathfrak{x}_{1}^{\prime},
$$

where $u_{2}$ denotes the affine curve length at $\mathfrak{r}_{2}$.

Hence the relations become

$$
\begin{gathered}
\frac{\mathfrak{x}_{2}^{\prime}}{\left|\mathfrak{x}_{2}^{\prime}\right|}=-\frac{\mathfrak{x}_{1}^{\prime}}{\left|\mathfrak{x}_{1}^{\prime}\right|}, \quad \mathfrak{x}_{1}^{\prime \prime \prime}=-k_{1} \mathfrak{c}_{1}^{\prime},\left(^{3}\right) \\
\mathfrak{x}_{2}^{\prime \prime \prime}=-k_{2} \mathfrak{x}_{2}^{\prime}=k_{2} \frac{\left|\mathfrak{x}_{2}^{\prime}\right|}{\left|\mathfrak{x}_{1}^{\prime}\right|} \mathfrak{c}_{1}^{\prime} .
\end{gathered}
$$

From (5) the equation (4) takes the form

$$
\left\{1+\frac{\left|\mathfrak{x}_{2}^{\prime}\right|}{\left|\mathfrak{x}_{1}^{\prime}\right|} \frac{d u_{2}}{d u_{1}}-\frac{b}{2}\left(k_{1}+\frac{\left|\mathfrak{x}_{2}^{\prime}\right|}{\left|\mathfrak{x}_{1}^{\prime}\right|} \frac{d u_{2}}{d u_{1}}\right)-\sigma^{\prime}\right\} \mathfrak{x}_{1}^{\prime}=\sigma \mathfrak{x}_{1}^{\prime \prime}
$$

By means of $\left(x_{1}^{\prime}, x_{1}^{\prime \prime}\right)=1\left({ }^{3}\right)$

$$
\sigma=0
$$

Therefore the relation holds

$$
\mathfrak{x}_{1}-\mathfrak{x}_{2}+\frac{b}{2}\left(\mathfrak{x}_{1}^{\prime \prime}-\mathfrak{x}_{2}^{\prime \prime}\right)=0
$$

This equation shows that the vector $\mathfrak{x}_{1}-\mathfrak{x}_{2}$ is parallel to the vector $x_{1}^{\prime \prime}-\mathfrak{x}_{2}^{\prime \prime}$.

Next we prove that it is sufficient. If the two vectors are parallel, from (3) we get (6). By differentiation of (6) with raspect to $u_{1}$ we have

$$
\mathfrak{x}_{1}^{\prime}-\mathfrak{x}_{2}^{\prime} \frac{d u_{2}}{d u_{1}}+\frac{b}{2}\left(\mathfrak{x}_{1}^{\prime \prime \prime}-\mathfrak{x}_{2}^{\prime \prime \prime} \frac{d u_{2}}{d u_{1}}\right)+\frac{b}{2}\left(\mathfrak{x}_{1}^{\prime \prime}-\mathfrak{x}_{2}^{\prime \prime}\right)=0 \text {. }
$$

By means of (5) we get

$$
b^{\prime}=0, \quad \text { or } \quad b=\text { constant。 }
$$


Theorem 3. If $b$ is constant, then the following relation holds

$$
b=2 \frac{\bar{\rho}_{1}+\bar{\rho}_{2}}{k_{1} \bar{\rho}_{1}+k_{2} \bar{\rho}_{2}} .
$$

Proof. By differentiation of $(6)$ with respect to $u_{1}$

$$
1+\frac{\left|\mathfrak{c}_{2}^{\prime}\right|}{\left|\mathfrak{c}_{1}^{\prime}\right|} \frac{d u_{2}}{d u_{1}}-\frac{b}{2}\left(k_{1}+k_{2} \frac{\left|x_{2}^{\prime}\right|}{\left|\mathfrak{x}_{1}^{\prime}\right|} \frac{d u_{2}}{d u_{1}}\right)=0
$$

Hence $\left|x_{i}^{\prime}\right|=\bar{\rho}_{i}^{\frac{1}{3}}, \frac{d u_{i}}{d \varphi}=\bar{\rho}_{i}^{\frac{2}{3}}$ and then $\frac{d u_{2}}{d u_{1}}=\frac{\bar{\rho}_{2}^{\frac{2}{3}}}{\bar{\rho}_{1}^{\frac{2}{3}}}$.

Therefore the relation (7) becomes

$$
1+\frac{\bar{\rho}_{2}}{\bar{\rho}_{1}}-\frac{b}{2}\left(k_{1}+k_{2} \frac{\bar{\rho}_{2}}{\bar{\rho}_{1}}\right)=0,
$$

then the relation

$$
b=2 \frac{\bar{\rho}_{1}+\bar{\rho}_{2}}{k_{1} \bar{\rho}_{1}+k_{2} \bar{\rho}_{2}}
$$

holds.

Theorem 4. If the affine normals at the points of contact of two tangents are affine double normals of an oval, the oval is an ellipse and $b=\frac{2}{k}$.

Proof. From assumption it can be put

$$
\mathfrak{x}_{2}^{\prime \prime}=-\sigma_{1}^{\prime \prime}
$$

where $\sigma$ is a positive scalar quantity.

Then we have

$$
\mathfrak{x}_{2}^{\prime \prime \prime} \frac{d u_{2}}{d u_{1}}=-\sigma \mathfrak{x}_{1}^{\prime \prime \prime}-\sigma^{\prime} \mathfrak{d}_{1}^{\prime \prime}
$$

By means of (5) and $\left(\mathfrak{x}_{1}^{\prime}, \mathrm{c}_{1}^{\prime \prime}\right)=1, \sigma=$ constant and then $\sigma=1$.

Therefore

$$
\mathfrak{c}_{1}^{\prime \prime}=-\mathfrak{x}_{2}^{\prime \prime} .
$$

By means of (5) we have

therefore

$$
1=-\left(\mathfrak{x}_{1}^{\prime}, \mathfrak{x}_{1}^{\prime \prime}\right)=\frac{\bar{\rho}_{1}^{\frac{1}{3}}}{\bar{\rho}_{2}^{\frac{1}{3}}}\left(\mathfrak{x}_{2}^{\prime}, \mathfrak{c}_{2}^{\prime \prime}\right)=\frac{\bar{\rho}_{1}^{\frac{1}{3}}}{\bar{\rho}_{2}^{\frac{1}{3}}}
$$

$$
\bar{\rho}_{1}=\bar{\rho}_{2} \text {. }
$$


Then we have

$$
k_{1}=k_{2} \text {. }
$$

By the assumption the two vectors $\mathfrak{x}_{1}-\mathfrak{x}_{2}$ and $\mathfrak{x}_{1}^{\prime \prime}-\mathfrak{r}_{2}^{\prime \prime}$ are parallel. Therefore from Theorem 2 the oval must be of affine constant breadth. Then we obtain from Theorem 3, by means of (10) and (11),

$$
b=\frac{2}{k_{1}} \text {. }
$$

Then since $k$ is constant, the oval is an ellipse.

$\S 2$. The relation between the affine breadth and the $r$-breadth $\left({ }^{2}\right)$ of the oval.

Let us take the negative indicatrix of the affine curvature as the unit oval in the relative differential geometry $\left({ }^{4}\right)$, that is, put

$$
\mathfrak{e}=-\mathfrak{x}^{\prime \prime} \text {. }
$$

Then the relation holds

$$
b=2 \frac{\left(\left(\mathfrak{x}_{2}-\mathfrak{x}_{1}\right) \xi_{1}\right)}{\left(\left(\mathfrak{x}_{1}^{\prime \prime}-\mathfrak{x}_{2}^{\prime \prime}\right) \xi_{1}\right)}=2 \frac{\left(\left(\mathfrak{x}_{1}-\mathfrak{x}_{2}\right) \xi_{1}\right)}{\left(\left(\mathfrak{e}_{1}-\mathfrak{e}_{2}\right) \xi_{1}\right)}=B,\left({ }^{2}\right)
$$

or

$$
b=B \text {. }
$$

Therefore Theorem 2 and Theorem 3 are obtained from the corresponding theorems in my preceding paper.

\section{§3. The affine breadth of the ovaloid.}

Let $\mathfrak{y}_{1}$ and $\mathfrak{y}_{2}$ be respectively the affine normals at the points of contact $\mathfrak{x}_{1}$ and $\mathfrak{x}_{2}$ of the parallel tangent planes on the ovaloid $\mathfrak{x}_{\text {. }}$ Let us put

$$
b=2 \frac{\left(\left(\mathfrak{x}_{2}-\mathfrak{x}_{1}\right) \xi_{1}\right)}{\left(\mathfrak{y}_{1} \xi_{1}\right)+\left(\mathfrak{y}_{2} \xi_{2}\right)},
$$

where $b$ is defined as the affine breadth.

Hence we have

$$
\mathfrak{y}=G^{m l}\left(\frac{\partial}{\partial u^{l}} \log \left|\overline{K^{\prime}}\right|^{-\frac{1}{4}}\right) \mathfrak{x}_{m}+|\bar{K}|^{\frac{1}{4}} \xi,\left(^{3}\right)
$$

(4) W. Süss, Zur relativen Differentialgeometrie I. Japanese Journ. of Math., 4 (1927)。 
then since $\bar{K}>0,\left(\mathfrak{y}_{i} \xi_{i}\right)=\bar{K}^{\frac{1}{4}}$.

Therefore we have

$$
b=2 \frac{p_{1}+p_{2}}{\bar{K}_{1}^{\frac{1}{4}}+\bar{K}_{2}^{\frac{1}{4}}}
$$

Theorem 1. Let $\mathfrak{p}_{i}$ denote the affine distance from $\mathfrak{x}_{i}$ to $x_{k}(i, k=1,2$; $i \neq k$ ), then $b$ is equal to the harmonic mean of $p_{1}$ and $\mathfrak{p}_{2}$, and then $b$ is an affine invariant.

Proof. By assumption we have

$$
p_{1}=\bar{K}_{1}^{-\frac{1}{4}}\left(\left(x_{2}-x_{1}\right) \xi_{1}\right), \quad p_{2}=\bar{K}_{2}^{-\frac{1}{4}}\left(\left(x_{1}-x_{2}\right) \xi_{2}\right) .
$$

From the definition of $b$

$$
\begin{aligned}
\frac{2}{b} & =\frac{\bar{K}_{1}^{\frac{1}{4}}+\bar{K}_{2}^{\frac{1}{4}}}{\left(\left(\mathfrak{x}_{2}-\mathfrak{x}_{1}\right) \xi_{1}\right)} \\
& =\frac{1}{\bar{K}_{1}^{-\frac{1}{4}}\left(\left(\mathfrak{x}_{2}-\mathfrak{x}_{1}\right) \xi_{1}\right)}+\frac{1}{\bar{K}_{2}^{-\frac{1}{4}}\left(\left(\mathfrak{x}_{1}-\mathfrak{x}_{2}\right) \xi_{2}\right)} \\
& =\frac{1}{p_{1}}+\frac{1}{\mathfrak{p}_{2}} .
\end{aligned}
$$

Theorem 2. A necessary and sufficient condition that the affine breadth of an ovaloid $E(x)$ be constant, is that the two vectors $x_{1}-x_{2}$ and $\mathfrak{y}_{1}-\mathfrak{y}_{2}$ are parallel.

Proof. From (1) we have

$$
\left(\left\{\mathfrak{x}_{1}-\mathfrak{x}_{2}+\frac{b}{2}\left(\mathfrak{y}_{1}-\mathfrak{y}_{2}\right)\right\} \xi_{1}\right)=0 \text { 。 }
$$

Therefore it can be put

$$
\mathfrak{x}_{1}-\mathfrak{x}_{2}+\frac{b}{2}\left(\mathfrak{y}_{1}-\mathfrak{y}_{2}\right)=\alpha x_{1 u}+\beta \mathfrak{x}_{1 v} .
$$

By differentiation of (4) with respect to the parameters $u$ and $v$

$$
\begin{aligned}
& x_{1 u}-\mathfrak{x}_{2 u}+\frac{b}{2}\left(\mathfrak{y}_{1 u}-\mathfrak{y}_{2 u}\right)=\alpha x_{1 u u}+\beta x_{1 u v}+\alpha_{u} \mathfrak{x}_{1 u}+\beta_{u} \mathfrak{x}_{1 v}, \\
& \mathfrak{x}_{1 v}-\mathfrak{x}_{2 v}+\frac{b}{2}\left(\mathfrak{y}_{1 v}-\mathfrak{y}_{2 v}\right)=\alpha x_{1 u v}+\beta x_{1 v v}+\alpha_{v} \mathfrak{x}_{1 u}+\beta_{v} \mathfrak{x}_{1 v} .
\end{aligned}
$$


By means of the relation $\mathfrak{y}_{1 i}=B_{i}^{k} \mathfrak{X}_{1 k},\left({ }^{3}\right)$ in the same manner as in the proof of the corresponding theorem in my preceding paper, $\left({ }^{2}\right)$ we have

$$
\alpha=\beta=0 \text {. }
$$

Conversely it can be easily proved that it is sufficient.

Theorem 3. If $b$ be constant, then we have

$$
b=2 \frac{\left(D_{1}+D_{2}\right) R_{1}^{1} R_{1}^{2}}{D_{1} R_{1}^{2}+D_{2} R_{1}^{1}}=2 \frac{\left(D_{1}^{\prime \prime}+D_{2}^{\prime \prime}\right) R_{2}^{1} R_{2}^{2}}{D_{1}^{\prime \prime} R_{2}^{2}+D_{2}^{\prime \prime} R_{2}^{1}}
$$

where $D_{i}=\left(\Upsilon_{i u n} \xi_{i}\right), D_{i}^{\prime \prime}=\left(\Upsilon_{i v v} \xi_{i}\right)$ and $R_{1}^{i}$ and $R_{2}^{i}$ are the radii of the affine curvature at $\mathrm{x}_{i}$.

Proof. Since $b$ is constant, by Theorem 2 we obtain

$$
\mathfrak{x}_{1}-\mathfrak{x}_{2}+\frac{b}{2}\left(\mathfrak{y}_{1}-\mathfrak{y}_{2}\right)=0 \text {. }
$$

Let the lines of affine curvature be taken as parameter-curves, so that

$$
\left\{\begin{array}{l}
\mathfrak{x}_{i u}=-R_{1}^{i} \mathfrak{y}_{i u},\left({ }^{3}\right) \\
\mathfrak{x}_{i v}=-R_{2}^{i} \mathfrak{y}_{i v} .
\end{array}\right.
$$

By means of (6) and (7)

$$
\begin{aligned}
& \left(1-\frac{b}{2 R_{1}^{1}}\right) x_{1 u}=\left(1-\frac{b}{2 R_{1}^{2}}\right) x_{2 u}, \\
& \left(1-\frac{b}{2 R_{2}^{1}}\right) x_{1 v}=\left(1-\frac{b}{2 R_{2}^{2}}\right) x_{2 v} .
\end{aligned}
$$

Let us differentiate (8) respectively with respect to $u$ and $v$, and let us form the scalar product of both sides of the obtained equation with $\xi_{1}=-\xi_{2}$, so that we have

$$
\begin{aligned}
& \left(1-\frac{b}{2 R_{1}^{1}}\right) D_{1}=-\left(1-\frac{b}{2 R_{1}^{2}}\right) D_{2} \\
& \left(1-\frac{b}{2 R_{2}^{1}}\right) D_{1}^{\prime \prime}=-\left(1-\frac{b}{2 R_{2}^{2}}\right) D_{2}^{\prime \prime}
\end{aligned}
$$

Therefore the relation (5) holds.

Theorem 4. If the affine normals at the points of contact of the parallel tangent planes on $E(x)$ are an affine double normal, the ovaloid is an ellipsoid, and the relation $\frac{b}{2}=R_{1}=R_{2}=$ constant follows. 
Proof. By assumption and Theorem 2 we have

$$
b=\text { constant }
$$

and then it can be put

$$
\mathfrak{r}_{1}-\mathfrak{r}_{2}=-\frac{b}{2}\left(\mathfrak{y}_{1}-\mathfrak{y}_{2}\right) \equiv \lambda \mathfrak{y}_{1} .
$$

Then

$$
\left(\lambda+\frac{b}{2}\right) \mathfrak{y}_{1}=\frac{b}{2} \mathfrak{y}_{2}
$$

By differentiation of (ii) we get

$$
\begin{aligned}
& \lambda_{u} \mathfrak{y}_{1}+\left(\lambda+\frac{b}{2}\right) \mathfrak{y}_{1 u}=\frac{b}{2} \mathfrak{y}_{2 u}, \\
& \lambda_{v} \mathfrak{y}_{2}+\left(\lambda+\frac{b}{2}\right) \mathfrak{y}_{1 v}=\frac{b}{2} \mathfrak{y}_{2 v} .
\end{aligned}
$$

Let us form the scalar product of both members of the above equation with $\xi_{1}=-\xi_{2}$, so that the relation $\lambda_{u}=\lambda_{v}=0$ follows, or $\lambda=$ constant. From (ii) $\lambda=-b$, and then

$$
\mathfrak{y}_{1}+\mathfrak{y}_{2}=0 \text { 。 }
$$

By means of (iii) and the relation $\xi_{1}=-\xi_{2}$ we get

$$
\bar{K}_{1}=\bar{K}_{2} \text { 。 }
$$

Therefore, by Prof. T. Kubota's theorem $\left(^{5}\right) E(x)$ must be central. And then from (1) we have

$$
\mathfrak{x}_{1}=-\frac{b}{2} \mathfrak{y}_{1}
$$

Let us take the lines of affine curvature as parameter-curves, then we obtain (7). By the relation (7) we have

$$
R_{1}^{1}=R_{2}^{1}=\frac{b}{2}=\text { constant }
$$

Sinec $H=\frac{1}{2}\left(\frac{1}{R_{1}^{1}}+\frac{1}{R_{2}^{1}}\right)=$ const., the ovaloid is an ellipsoid. $\left({ }^{3}\right)$

(5) Einige Probleme über konvex-geschlossene Kurven und F'lächen; Tôhoku Math. Journ, 17 (1920). 
$\S 4$. The relation between the affine breadth and the $r$-breadth of the ovaloid.

Let us take the negative indicatrix of the affine curvature as the unit ovaloid in the relative differential geometry, that is, put $e=-\mathfrak{y}$. Then the relation holds

$$
b=2 \frac{\left(\left(\mathfrak{x}_{2}-\mathfrak{r}_{1}\right) \xi_{1}\right)}{\left(\mathfrak{y}_{1} \xi_{1}\right)+\left(\mathfrak{y}_{2} \xi_{2}\right)}=2 \frac{\left.\left(\mathfrak{k}_{1}-\mathfrak{x}_{2}\right) \xi_{1}\right)}{\left(\left(\mathfrak{e}_{1}-\mathfrak{e}_{2}\right) \xi_{1}\right)}=B,\left({ }^{2}\right)
$$

or

$$
b=B \text {. }
$$

Theorem 2 and Theorem $\mathscr{3}$ can be deduced from the corresponding theorems in my preceding paper. $\left({ }^{2}\right)$ 